\title{
Clinical efficacy and mechanism of mesenchymal stromal cells in treatment of COVID-19
}

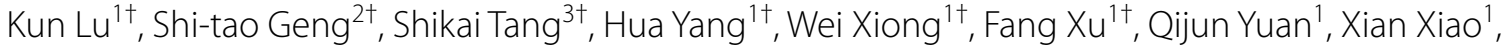 \\ Renqiang Huang ${ }^{1}$, Haihui Liang ${ }^{1}$, Zhipeng Chen ${ }^{1}$, Chuanyun Qian ${ }^{3^{*}}$, Yang Li ${ }^{1 *} \mathbb{D}$ and Songqing Wang ${ }^{1 *}$
}

\begin{abstract}
Coronavirus disease 2019 (COVID-19) is a highly infectious epidemic disease that has seriously affected human health worldwide. To date, however, there is still no definitive drug for the treatment of COVID-19. Cell-based therapies could represent a new breakthrough. Over the past several decades, mesenchymal stromal cells (MSCs) have proven to be ideal candidates for the treatment of many viral infectious diseases due to their immunomodulatory and tissue repair or regeneration promoting properties, and several relevant clinical trials for the treatment of COVID-19 have been registered internationally. Herein, we systematically summarize the clinical efficacy of MSCs in the treatment of COVID-19 based on published results, including mortality, time to symptom improvement, computed tomography (CT) imaging, cytokines, and safety, while elaborating on the possible mechanisms underpinning the effects of MSCs, to provide a reference for subsequent studies.
\end{abstract}

Keywords: Coronavirus disease 2019, Mesenchymal stromal cells, Clinical efficacy, Immunomodulation, Tissue repair

\section{Introduction}

Coronavirus disease 2019 (COVID-19) is a highly infectious disease caused by severe acute respiratory syndrome coronavirus 2 (SARS-CoV-2). Based on available data, one of the hallmarks of SARS-CoV-2 infection pathogenesis is cytokine storm in the lung. Acute virusinduced cytokine release can lead to pulmonary edema, ventilatory dysfunction, and acute respiratory distress syndrome (ARDS) [1], especially in severe cases, which

\footnotetext{
*Correspondence: qianchuanyun@126.com; 283105164@qq.com; wangsongqing2004@126.com

${ }^{\dagger}$ Kun Lu, Shi-tao Geng and Shikai Tang are co-first author and contributed equally to this work

${ }^{\dagger}$ Hua Yang, Wei Xiong and Fang Xu are co-second author and contributed equally to this work

1 Department of Neurosurgery, First Naval Hospital of Southern Theater Command, Zhanjiang 524000, China

${ }^{3}$ Department of Emergency, First Affiliated Hospital of Kunming Medical University, Kunming 650032, China

Full list of author information is available at the end of the article
}

is characterized by the up-regulation of proinflammatory cytokines and chemokines, abnormal cellular immune response, respiratory and cardiovascular failure, end organ injury, and possibly death [2, 3]. To date, however, no specific antiviral drug has been proven effective in the treatment of COVID-19 and therefore is urgently required. A variety of strategies have been proposed to control cytokine storms and reduce mortality, such as selective cytokine blockade (e.g., anakinra or tocilizumab), JAK inhibition, intravenous immunoglobulin administration, mesenchymal stromal cell (MSC) therapy, and artificial blood purification $[4,5]$.

Based on the results of several models and stageclinical trials of ARDS and sepsis, cell-based therapy, especially stem cell therapy, has emerged as a promising therapeutic area, especially for the treatment of COVID-19 [6-8]. MSCs are pluripotent cells obtained from different tissues, such as adipose and bone marrow. Due to their immunomodulatory properties, original author(s) and the source, provide a link to the Creative Commons licence, and indicate if changes were made. The images or other third party material in this article are included in the article's Creative Commons licence, unless indicated otherwise in a credit line to the material. If material is not included in the article's Creative Commons licence and your intended use is not permitted by statutory regulation or exceeds the permitted use, you will need to obtain permission directly from the copyright holder. To view a copy of this licence, visit http://creativecommons.org/licenses/by/4.0/. The Creative Commons Public Domain Dedication waiver (http://creativecommons.org/publicdomain/zero/1.0/) applies to the data made available in this article, unless otherwise stated in a credit line to the data. 
MSCs can alter immune cell function, modulate immune responses, and reduce inflammation-induced lung injury [9]. These cells can also prevent apoptosis and regenerate lung cells, especially type II alveolar cells, by producing growth factors such as keratinocyte growth factor (KGF), vascular endothelial growth factor (VEGF), and hepatocyte growth factor (HGF) [10]. Furthermore, by expressing indoleamine 2,3-dioxygenase (IDO), MSCs can stimulate interferon- $\gamma$ (IFN- $\gamma$ ) production and IFN- $\gamma$-independent restriction of viral replication $[11,12]$. Thus, the immunomodulatory, tissue repair, and antiviral properties of MSCs highlight their potential for the treatment of COVID-19 [10].

The safety and efficacy of MSCs have been demonstrated in multiple clinical trials related to the treatment of COVID-19 [6-8, 13]. As of October 2021, 51clinical trials for MSC-based COVID-19 therapy have been registered (http://clinicaltrials.gov/ and http://www.chictr.org.cn/) (Table 1). In this article, we summarize the findings of seven published clinical trials (Table 2) to help clarify the regulation of cytokines by MSCs in patients with COVID-19, and whether this therapy can improve patient symptoms, shorten hospital stays, and reduce mortality, in conjunction with a high safety and low adverse event profile. The underlying mechanisms by which MSCs may act are also explored, with the view to provide a reference for subsequent trials and research.

\section{Efficacy \\ Mortality}

According to the World Health Organization (WHO), the cumulative number of deaths due to COVID-19 has surpassed 5.5 million globally. Multiple studies have shown that MSC therapy can significantly reduce the incidence and mortality of critical illness (Table 3) [14-19]. For example, Shu et al. [14] found the mortality in the MSC treatment group was zero. In addition, $\mathrm{Xu}$ et al. [17] reported significantly higher survival in patients treated with MSC (92.31\%) than those in the routine treatment group (66.67\%). Another study reported a 28-day survival rate of $91 \%$ in MSC-treated patients compared with $42 \%$ in non-treated controls, with the control group also showing a higher risk of death (hazard ratio (HR) 8.76; 95\% CI 1.07-71.4) [18]. Furthermore, serious adverse event-free survival showed significant improvement with MSC treatment compared with the controls (HR 6.22; 95\% CI 1.33-28.96). Thus, the above results confirm the safety of MSC therapy and its effectiveness at reducing mortality and improving survival. However, due to the small sample sizes in the above studies, data from largescale clinical phase 3 trials are still required.

\section{Systemic changes and symptoms}

MSC treatment can significantly shorten the time to clinical symptom improvement in COVID-19 patients. Lanzoni et al. [18] showed a significantly shorter COVID-19 recovery time following MSC treatment, with an HR for recovery of 0.29 (95\% CI 0.09-0.95) in the control group versus the MSC-treated group. Several other studies have also demonstrated that MSC therapy can significantly expedite patient recovery. A recent experiment comparing pulmonary function recovery and comprehensive reserve capacity based on a 6-min walk test (6-MWT) found that walk distance was longer in MSC-treated patients compared with the controls, although maximal forced vital capacity (VCmax), diffusing lung capacity for carbon monoxide (DLCO), six category scale, oxygen therapy status, and mMRC dyspnea score did not differ significantly between the two groups [20]. In critically ill patients, the partial pressure of arterial oxygen: percentage of inspired oxygen $\left(\mathrm{PaO}_{2} / \mathrm{FiO}_{2}\right)$ ratio showed improvement after MSC treatment [16]. Furthermore, MSC-treated patients demonstrated significant improvement in clinical symptoms and were discharged from the ICU within 2-7 days after MSC infusion, with significant relief of dyspnea, decrease in respiratory rate within 48-96 h, and improvement in oxygen saturation [15]. Shu et al. [14] also reported significant improvements in weakness, fatigue, shortness of breath, and low oxygen saturation in MSC-treated patients compared with the controls.

In addition to clinical symptoms, laboratory parameters have also been examined $[15,16]$, including C-reactive protein (CRP), alanine aminotransferase (ALT), creatinine, serum ferritin (SF), and platelet levels, which all returned to their normal range after MSC administration. These results suggest that MSCs not only improve pulmonary symptoms but also positively impact the functional recovery of multiple organs, such as the liver and kidney.

\section{Computed tomography (CT) imaging}

As clinical symptoms are influenced by multiple factors, and the evaluation of symptom relief is subjective, various researchers have assessed lung lesions in COVID-19 patients using imaging. Studies using CT imaging have reported that time of pulmonary lesions is significantly shortened in patients treated with MSCs. A single-center, open-label, randomized, standard treatment-controlled trial showed that CT score, number of lobes affected, ground glass opacity, and solid changes were significantly improved in MSC-treated patients compared with the controls [14]. In another study, the rate of chest imaging changes 1 month after MSC infusion was significantly 


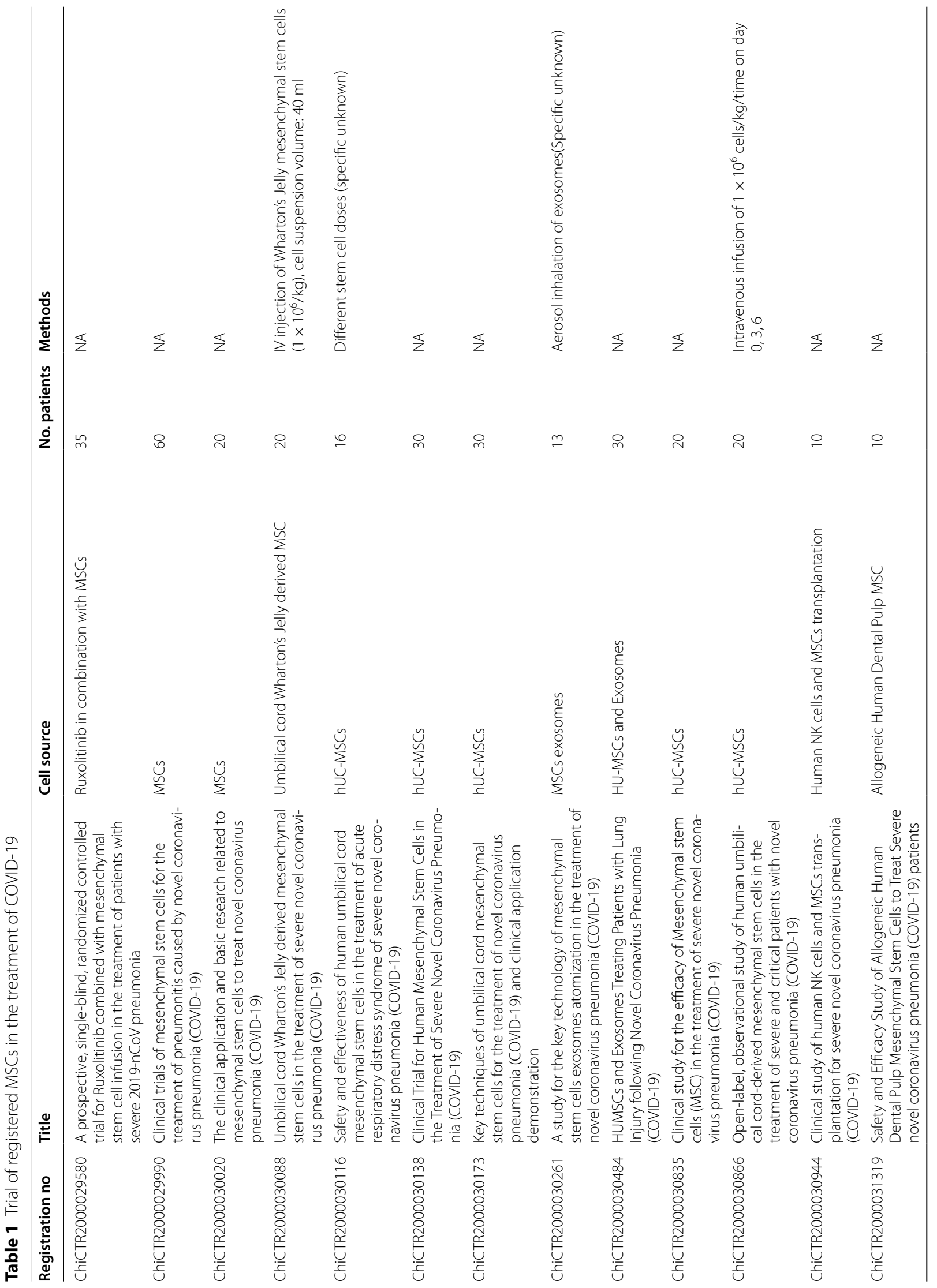




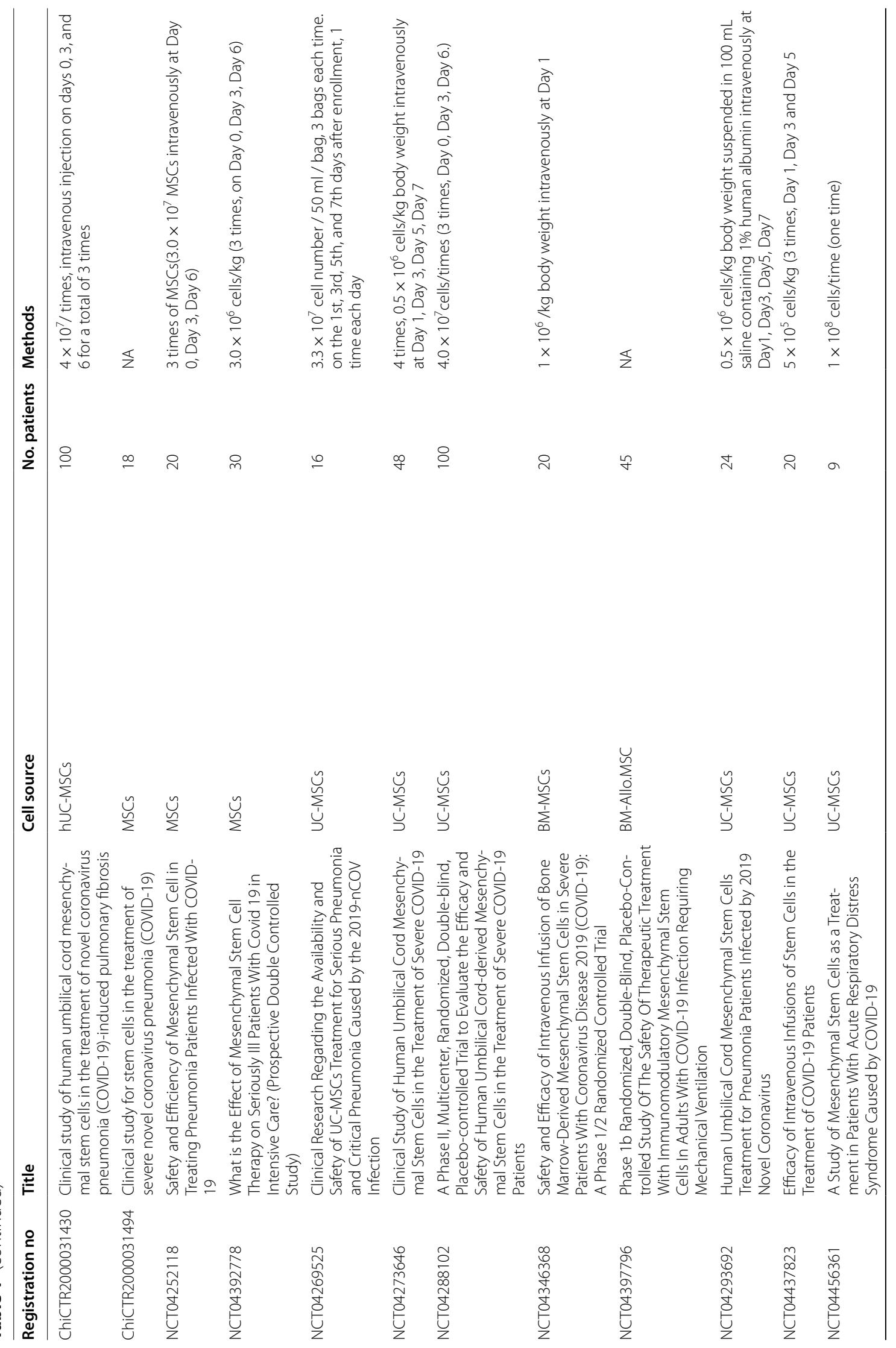




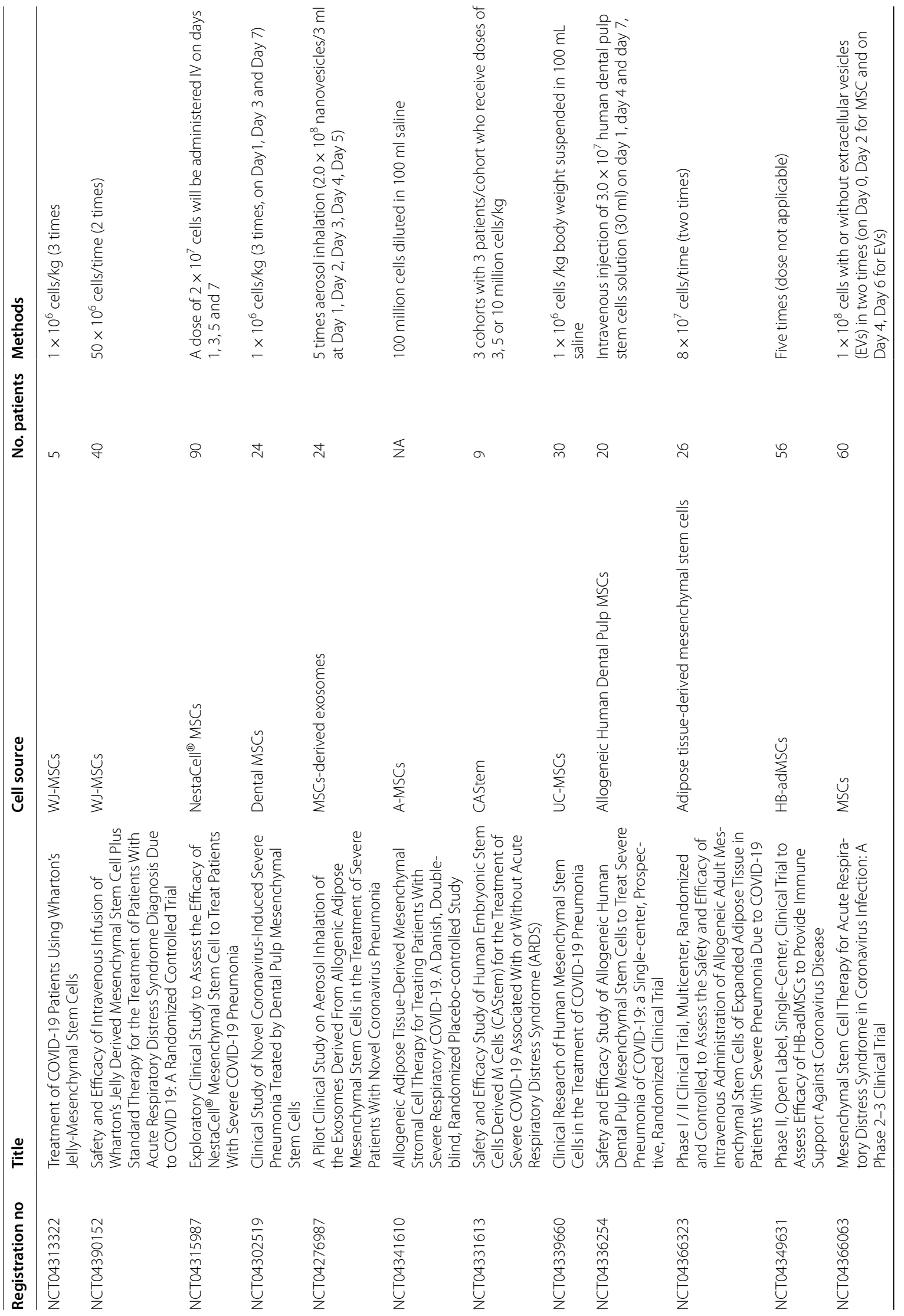




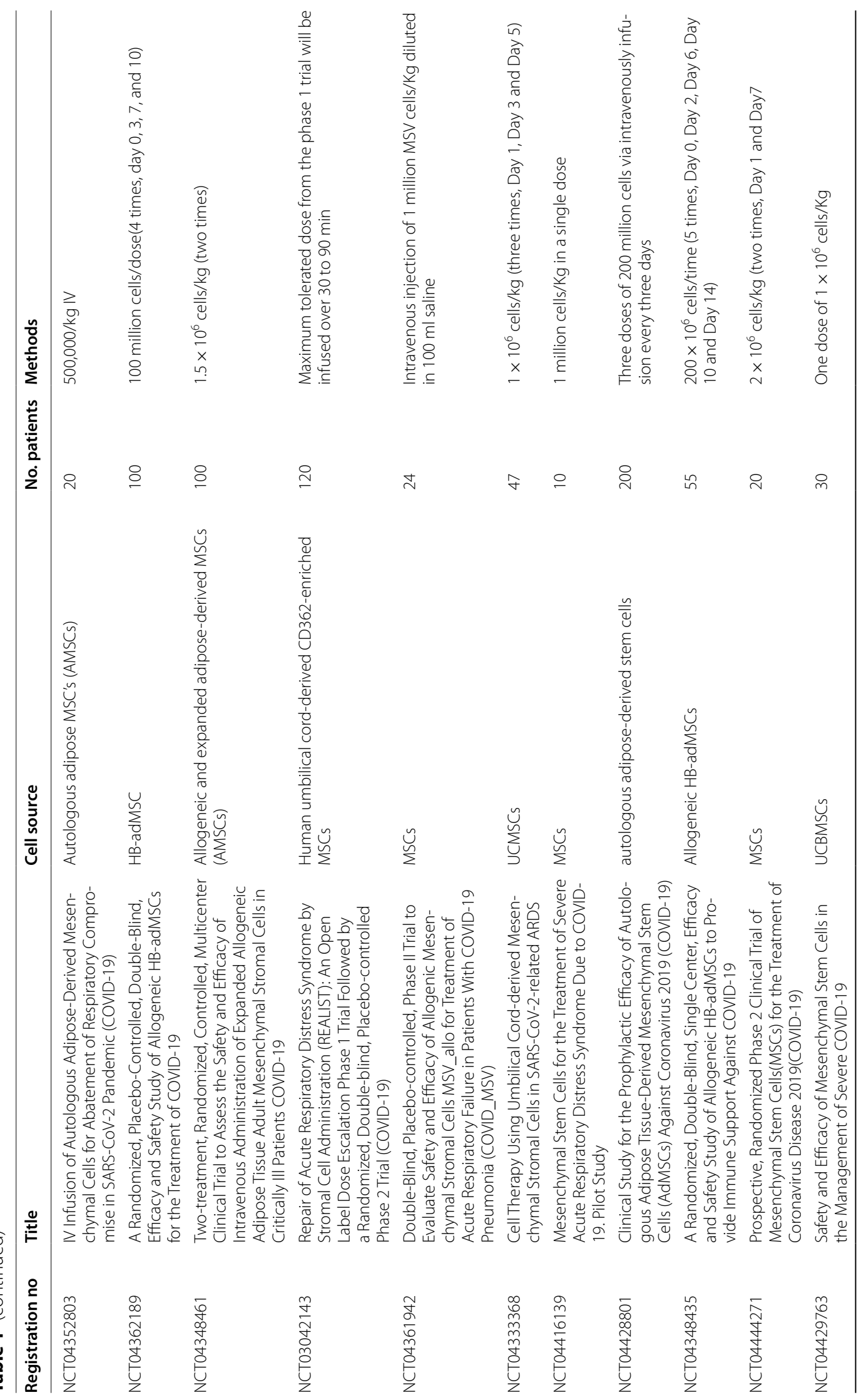




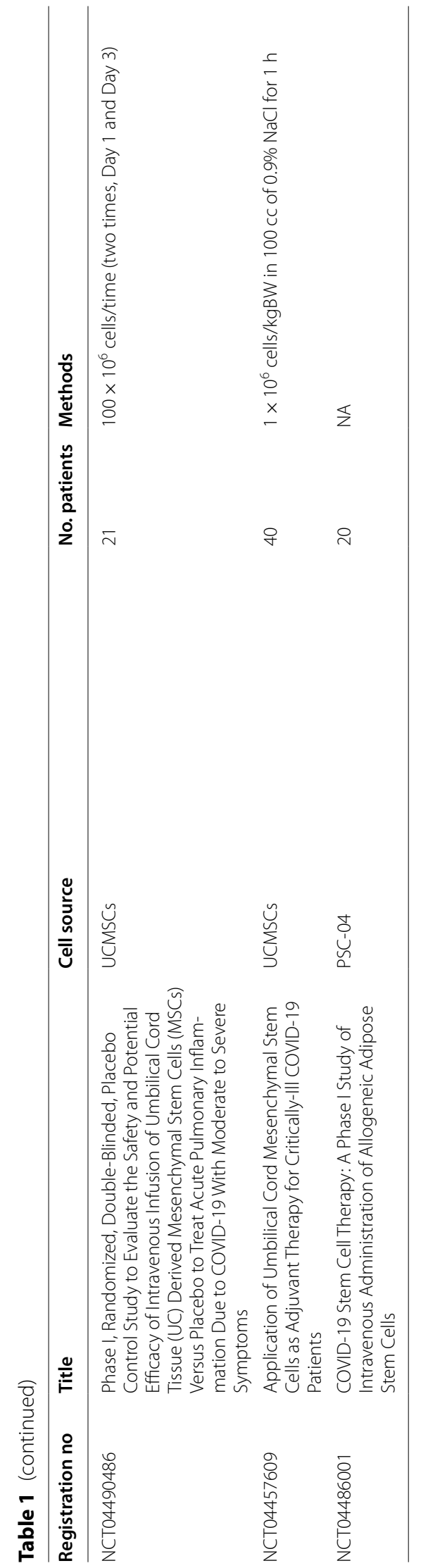




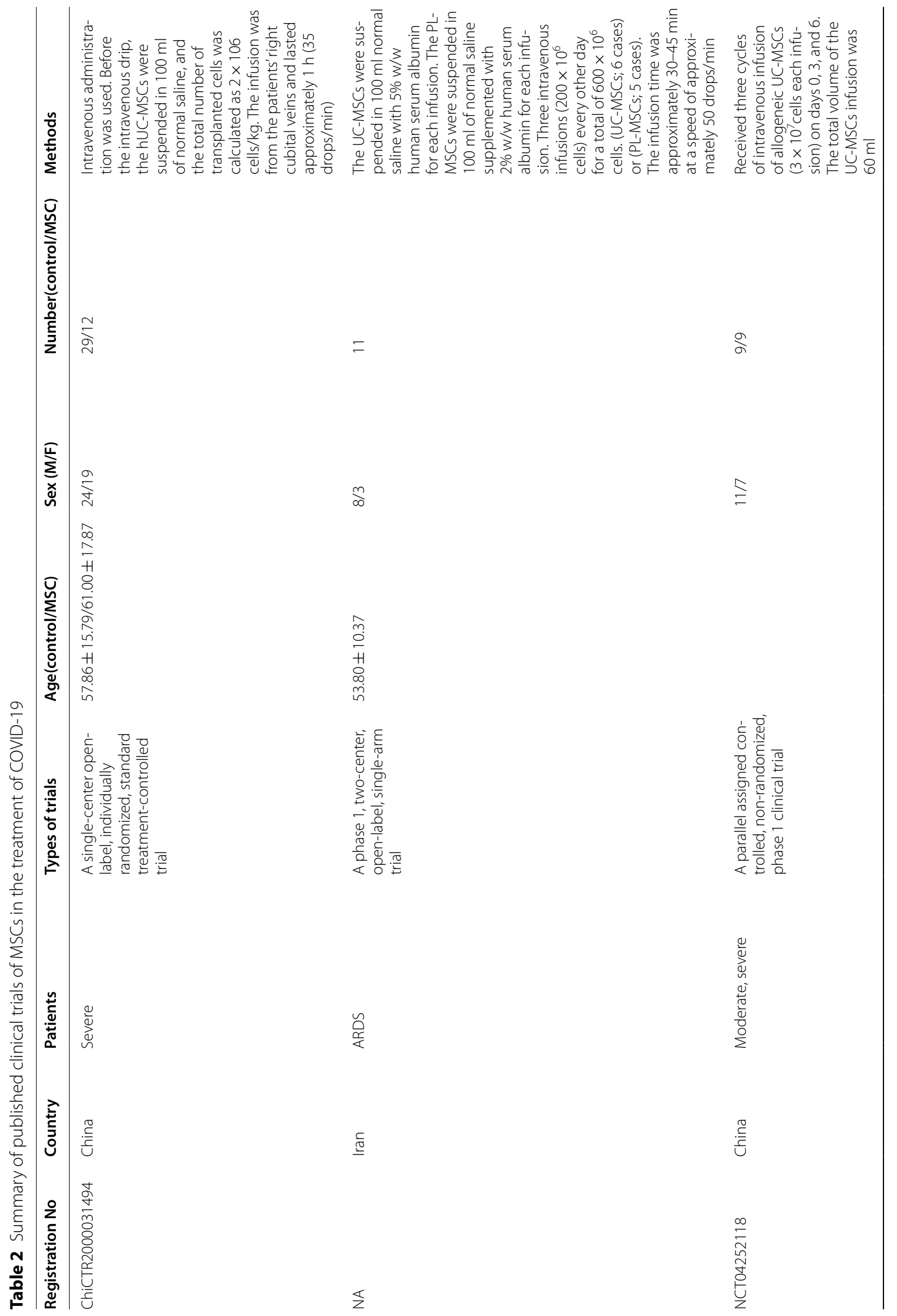




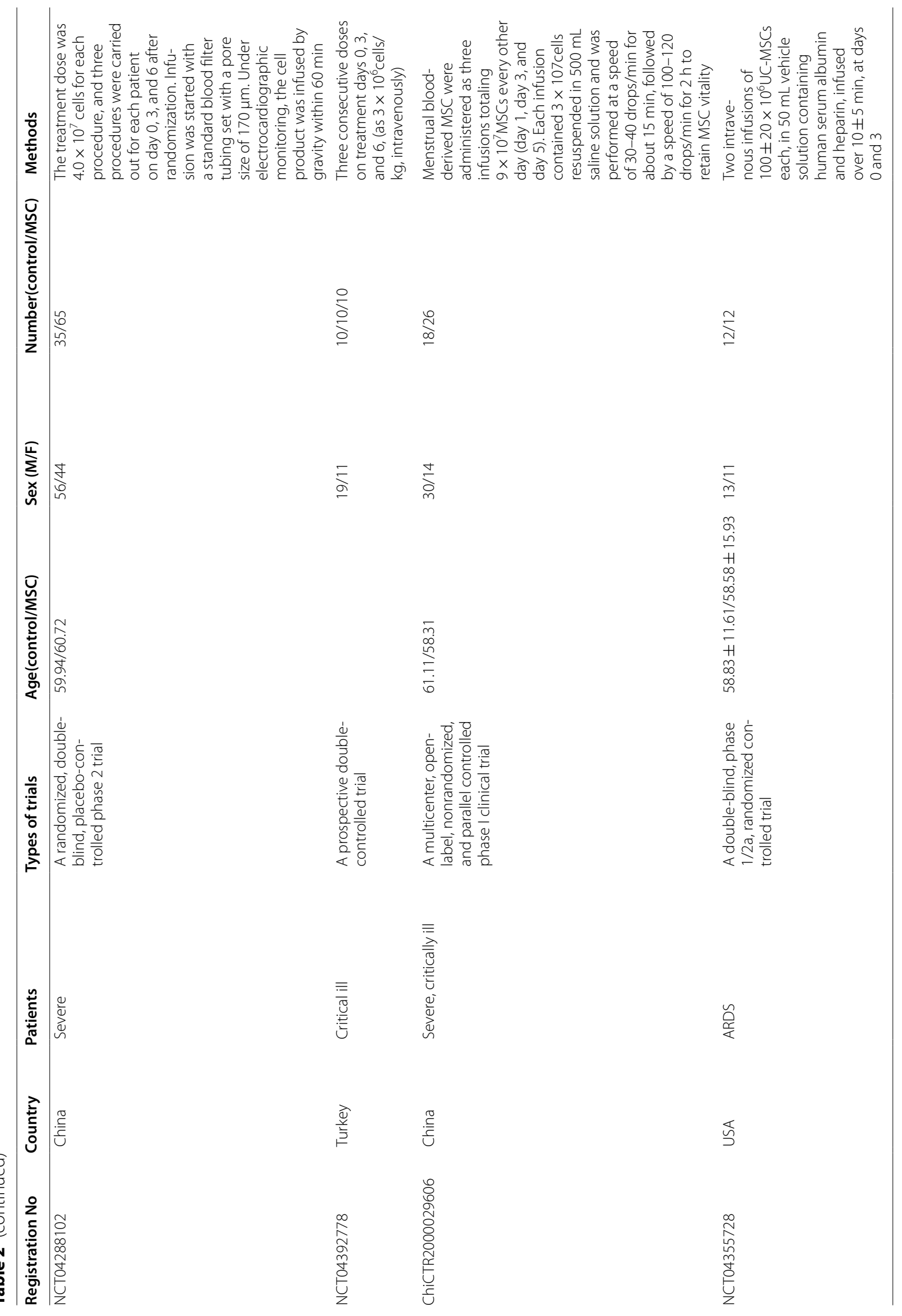




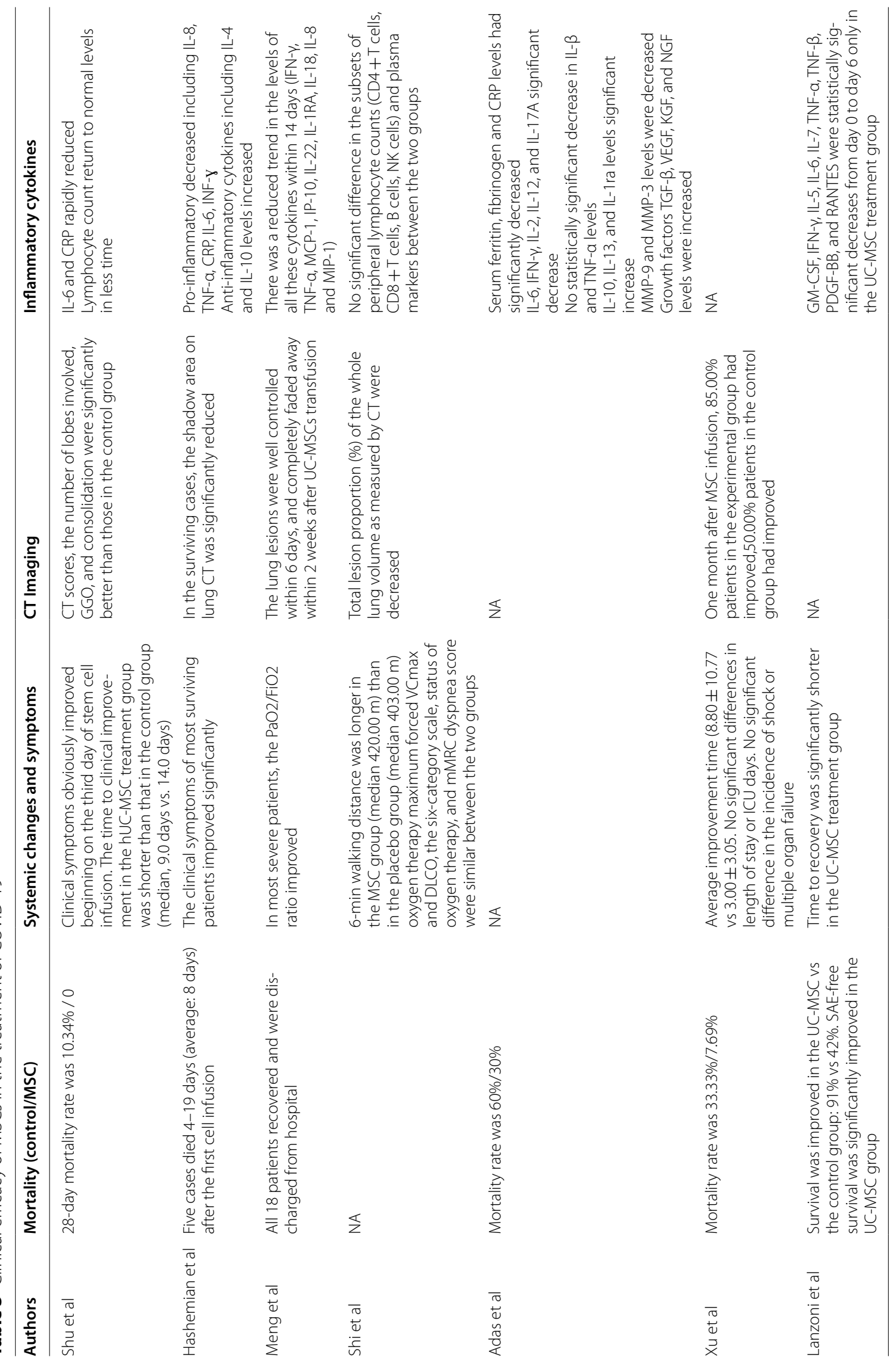


improved in $85 \%$ of MSC-treated patients compared to $50 \%$ of control-group patients [17]. Previous research also reported that lung lesions were well controlled within 6 days and completely disappeared within 2 weeks after MSC infusion [16]. A phase 2 clinical trial also reported a significant decrease in total lesion proportion in the whole lung measured by CT from baseline to day 28 after MSC infusion [20].

\section{Cytokines}

Cytokine storms are considered one of the main characteristics of COVID-19. To date, however, no definitive therapy has been shown to completely control cytokine storm or restore organ damage caused by infection with SARS-CoV-2. MSC transplantation may act as an immunomodulator in the development of cytokine storm caused by inflammation. Therefore, many scholars believe that MSCs have a decided advantage in controlling cytokine storm induced by COVID- 19 .

Several published clinical trials indicate that MSCs can effectively control the expression of inflammatory cytokines in COVID-19 patients [14-16, 18, 19]. Several studies have reported that proinflammatory cytokines, such as interleukin (IL)-8, tumor necrosis factor (TNF)- $\alpha$, CRP, IL-6, INF- $\gamma$, IL-2, IL-12, and IL-17A, decreased significantly after MSC infusion, while antiinflammatory cytokines IL-4 and IL-10 increased significantly $[14,15,18]$. However, other research reported that IL- $\beta$ and TNF- $\alpha$ levels were not significantly reduced after MSC administration [19]. In addition, a clinical trial reported no significant changes in cytokines, but a reduced trend in cytokine levels within 14 days (IFN- $\gamma$, TNF- $\alpha$, monocyte chemokine-1 (MCP-1), interferon inducible protein-10 (IP-10), IL-22, IL-1RA, IL-18, IL-8, and MIP-1) [16]. Intragroup analysis found that granulocyte macrophage colony stimulating factor (GM-CSF), IFN- $\gamma$, IL-5, IL-6, IL-7, TNF- $\alpha$, TNF- $\beta$, platelet derived growth factor-BB (PDGF-BB), and RANTES decreased significantly from days 0 to 6 in the UC-MSC treatment group [18]. These data, to some extent, demonstrate the effect of MSC treatment in patients with COVID-19, and the differences in outcomes between trials may be due to differences in disease degree and age. Thus, the results of large-scale phase 3 trials should help further define the role of MSCs in treatment.

\section{Safety}

Drug and treatment safety must be considered in clinical application. Recently, the clinical outcome of a 65-year-old female patient with COVID-19 treated with allogeneic human umbilical cord blood-derived MSCs (UCB-MSCs) was reported, with no adverse events noted during treatment and was well tolerated
[21]. Hashemian et al. also reported that the liver and kidney function of patients were not affected during MSC infusion [15]. Another study found that MSCs can cure or significantly improve the functional results of patients without obvious adverse events [22]. These results illustrate, to some extent, the safety of MSCs for the treatment of COVID-19, but the number of included experimenters is small and lack of randomized controlled trials. In view of this, many clinical trials have begun to evaluate the safety, feasibility, and tolerability of MSCs.

Various trials suggest that MSC therapy is safe for COVID-19. A prospective double-controlled trial reported no adverse or serious adverse events related to MSC treatment [19]. Another phase 1 clinical trial evaluated the safety of MSC infusion in patients with moderate to severe COVID-19 and reported that three patients experienced adverse effects, although these events were considered to be caused by disease progression based on pre-existing symptoms [16]. Similarly, two clinical randomized controlled trials $[18,20]$ showed that the overall incidence of adverse events was similar in the MSC and control groups, but these events were largely unrelated to treatment, and significantly more subjects experienced serious adverse events in the control group than in the MSC group. Thus, the above findings reflect the safety of MSC therapy and its potential benefits at reducing COVID-19-related adverse events.

In general, although the safety of MSCs is acceptable, treatment may not be applicable to some COVID-19 patients with serious complications. For example, a phase 1 trial [15] showed that multiple infusions of high-dose allogeneic MSCs were safe and rapidly improved respiratory distress and reduced inflammatory biomarkers in some cases of critically ill COVID-19-induced ARDS, but four patients with multiple organ failure or sepsis died within 5-19 days (mean 10 days) after the first MSC infusion. These findings suggest that contraindications need to be strictly considered when selecting patients for MSC therapy.

\section{Mechanisms of COVID-19 treatment with MSCs}

Several studies [23-27] have shown that MSCs can be safely infused intravenously or via the endobronchial route in humans, thus allowing MSCs to accumulate in the lungs to improve the lung microenvironment, protect alveolar epithelial cells, protect against pulmonary fibrosis, and improve lung function [28-30]. Although the specific molecular mechanisms underlying the effects of MSCs on COVID-19 treatment require further research, several studies have investigated possible processes, as summarized below. 


\section{Immunomodulation}

MSCs exhibit strong immunomodulatory potential. Their immunomodulatory functions are mainly exerted through cell-to-cell contact, paracrine secretion, endocrine action, and immune cell interactions (e.g., T cells, B cells, natural killer (NK) cells, macrophages, monocytes, dendritic cells (DCs), and neutrophils). Thus, MSCs participate in both innate and adaptive immunity [31-33], with regulatory $\mathrm{T}$ cell (Treg) and monocyte interactions appearing to play a key role [34]. Of course, it may vary according to the pathological mechanism of the disease, source of MSCs, and route of administration.

MSCs show immunosuppressive effects when exposed to sufficiently high levels of pro-inflammatory cytokines [35], but can promote an inflammatory response under low levels of TNF- $\alpha$ and IFN- $\gamma$ [35]. Thus, MSCs may need to be triggered by inflammatory cytokines to become immunosuppressive, and the inflammatory environment may be a key factor affecting immunoregulation of MSCs. One of the characteristics of COVID-19 is the formation of an inflammatory cytokine storm, which may provide an inflammatory environment for the immunomodulation of MSCs.

After infusion of MSCs in COVID-19 patients, IL1RA, IL-6, HGF, prostaglandin E2 (PGE2) secreted by MSCs promoted monocyte/macrophage differentiation into anti-inflammatory/immunomodulatory (type 2) phenotype, and directly inhibited differentiation into type 1 phenotype and DCs [36, 37]. On the one hand, type 2 monocytes/macrophages secrete high levels of IL-10, reduce expression levels of IL-12p70, TNF- $\alpha$, and IL-17, prevent monocytes from differentiating into DCs, and convert monocytes into an anti-inflammatory, IL10-secreting subtype via a positive feedback loop [36], while high levels of IL-10 inhibit T cell activity [38]. On the other hand, macrophages release more CCL-18 and transforming growth factor- $\beta 1$ (TGF- $\beta 1$ ) during differentiation into type 2 macrophages, which helps induce Treg formation, while CCL-18 converts memory $\mathrm{CD} 4^{+} \mathrm{T}$ cells into $\mathrm{CD} 4{ }^{+} \mathrm{CD} 25^{+} \mathrm{Foxp}^{+}$Treg cells and increases IL-10 and TGF- $\beta 1$ generation [39-41]. Furthermore, it was observed in the asthma model that the infused MSCs was engulfed by pulmonary macrophages, resulting in a shift of monocytes to a type 2 immunosuppressive phenotype, polarization of $\mathrm{CD} 14^{++} \mathrm{CD} 16^{-}$classical monocytes to a $\mathrm{CD} 14^{++} \mathrm{CD} 16^{+} \mathrm{CD} 206^{+}$immunoregulatory intermediate subset with anti-inflammatory properties, and increased expression of IL-10 and programmed death ligand-1 (PD-L1) [42-44], thereby driving the immune response toward an anti-inflammatory response.

MSCs act on the adaptive immune system, particularly $\mathrm{T}$ cells $[32,33]$, in various ways, e.g., inhibition of the proliferation, cytokine secretion, and cytotoxicity of $\mathrm{T}$ cells and regulation of T helper 1 (Th1)/T helper 2 (Th2) balance and Treg function. MSCs can induce IL-10 and PGE2 production and inhibit IL-17, IL-22, and IFN- $\gamma$ levels to limit Th17 differentiation [45] and suppress Th17 responses by modulating the IL-25/STAT3/PD-L1 axis [46]. Upon interaction with DCs, MSCs can cause a shift from pro-inflammatory Th1 cells to anti-inflammatory Th2 cells [47]. Induction of $\mathrm{CD} 4{ }^{+} \mathrm{CD} 25^{+}$Foxp $3^{+}$Tregs is one of the main features of MSC-mediated immune regulation, and MSCs can secrete TGF- $\beta 1$ and IDO to induce the formation of Tregs $[39,47]$. MSCs can directly interact with B cells and promote the generation of regulatory B cells (Bregs), which secrete IL-10 to convert effector $\mathrm{CD}^{+} \mathrm{T}$ cells into Foxp3 ${ }^{+}$Tregs $[48,49]$. MSCs can also exert direct immunosuppressive effects on $\mathrm{T}$ cell behavior by suppressing $\mathrm{CD}^{+} \mathrm{T}$ cell activation via the secretion of PD-L, including PD-L1 and PD-L2 [50, 51].

\section{Promotion of tissue repair and regeneration}

In addition to their immunomodulatory properties, the multilineage differentiation ability of MSCs also makes them ideal candidates for cell therapy. Numerous studies have demonstrated the regenerative capacity of MSCs in musculoskeletal system, nervous system, cardiac muscle, liver, cornea, trachea, and skin tissue repair [52].

COVID-19 is characterized by lung tissue damage, which can cause systemic multi organ damage, especially in patients who develop ARDS. After injection, MSCs can differentiate into lung tissue or secrete factors (e.g., angiopoietin-1 (ANGPT1), EGF, VEGF, PGE2, HGF, VEGFA, KGF, and IL-10) that can induce host repair/regenerative mechanisms [53], promote epithelial and endothelial repair, increase alveolar fluid clearance, regulate lung epithelial and endothelial permeability, and reduce inflammation in patients with ARDS lung injury [52, 54, 55]. In addition, these factors can promote tissue repair by supporting the growth and differentiation of local stem/ progenitor cells, regulating the deposition of extracellular matrix molecules, stimulating anti-scarring pathways, and inducing neovascularization [56, 57]. Induction factors secreted by MSCs, such as VEGF, brain-derived neurotrophic factor (BDNF), and TGF- $\beta 1$, can promote the development of self-repair. Activin A, EGF, KGF, HGF, and IGF-2 play important roles in MSC differentiation into epithelial cells by triggering appropriate signaling pathways [58, 59]. MSCs also show anti-apoptotic effects [53], and the release of KGF and HGF by MSCs can protect alveolar epithelial cells from apoptosis by increasing B-cell lymphoma-2 (BCL-2) expression and inhibiting hypoxia-inducible factor- $1 \alpha$ (HIF-1 $\alpha)$ protein expression [60]. Furthermore, the expression of various factors, such as VEGF, HGF, and TGF- $\beta$, can reverse endothelial cell apoptosis [61]. 
The immunomodulatory properties and tissue repair/ regeneration abilities of MSCs highlight their potential in the treatment of COVID-19. MSCs can also exert antibacterial effects by secreting soluble mediators to reduce the number of bacteria, improve the antibacterial response of immune cells, and inhibit the migration of proinflammatory cells into infected tissues [53]. Thus, MSCs can participate in the inhibition of viral replication via different mechanisms $[11,12]$.

\section{Conclusions}

As cells with multilineage differentiation ability, MSCs are potential candidates for cell-based therapy to treat COVID-19. Various clinical trials have demonstrated the efficacy and safety of MSCs for the treatment of COVID-19 patients, especially critically ill patients, not only improving clinical symptoms, hospital stay, cytokine release, and mortality, but also showing a high safety profile with limited adverse events. However, further evidence from large-scale and long-term phase 3 clinical trials is still required. Although the therapeutic action of MSCs in COVID-19 patients appears to involve immunomodulation, promotion of repair/regeneration, and inhibition of viral replication through cell-to-cell contact and paracrine activity, the specific mechanism needs further investigation. However, we expect more data from currently progressing trials should support the role of MSCs in COVID-19.

\begin{abstract}
Abbreviations
COVID-19: Coronavirus disease 2019; MSCs: Mesenchymal stromal cells; SARS-CoV-2: Severe acute respiratory syndrome coronavirus 2; ARDS: Acute respiratory distress syndrome; KGF: Keratinocyte growth factor; VEGF: Vascular endothelial growth factor; HGF: Hepatocyte growth factor; IDO: 2,3-Dioxygenase; IFN: Interferon; AE: Adverse event; 6-MWT: 6-Minute walk test; VCmax: Maximal forced vital capacity; DLCO: Diffusing lung capacity for carbon monoxide; $\mathrm{PaO}$ /FiO2: Pressure of arterial oxygen: percentage of inspired oxygen; CRP: C-reactive protein; ALT: Alanine aminotransferase; SF: Serum ferritin; GGO: Ground glass opacity; IL: Interleukin; TNF: Tumor necrosis factor; MCP-1: Monocyte chemokine-1; IP-10: Interferon-inducible protein-10; GM-CSF: Granulocyte macrophage colony stimulating factor; PDGF-BB: Platelet-derived growth factor-BB; NK: Natural killer; DCs: Dendritic cells; PGE2: Prostaglandin E2; TGF- $\beta 1$ : Transforming growth factor- $\beta 1$; PD-L1: Programmed death ligand-1; ANGPT1: Angiopoietin-1; EGF: Epidermal growth factor; BDNF: Brain-derived neurotrophic factor; BCL-2: B-cell lymphoma-2; HIF-1a: Hypoxiainducible factor-1a.
\end{abstract}

\section{Acknowledgements}

We gratefully acknowledge the assistance of Professor Haiying Wu in polishing the language of the article.

\section{Authors' contributions}

YL, SQW and CYQ conceptualized the outline and topic of the article. KL, STG and SKT participated in designing the study, drafting, writing and editing the manuscript. HY, WX, FX helped collect literature and draft manuscripts. QJY, XX made the form. RQH, HHL, ZPC participated in the revision of the manuscript. All authors read and approved the final manuscript.

\section{Funding}

There is no fund.
Availability of data and materials

Not applicable.

\section{Declarations}

Ethics approval and consent to participate

Not applicable.

\section{Consent for publication \\ Not applicable.}

\section{Competing interests}

The authors declare that they have no competing interests.

\section{Author details}

${ }^{1}$ Department of Neurosurgery, First Naval Hospital of Southern Theater Command, Zhanjiang 524000, China. ${ }^{2}$ Department of Emergency, First Naval Hospital of Southern Theater Command, Zhanjiang 524000, China. ${ }^{3}$ Department of Emergency, First Affiliated Hospital of Kunming Medical University, Kunming 650032, China.

Received: 20 December 2021 Accepted: 25 January 2022

Published online: 07 February 2022

\section{References}

1. Li H, Liu L, Zhang D, Xu J, Dai H, Tang N, et al. SARS-CoV-2 and viral sepsis: observations and hypotheses. Lancet. 2020;395(10235):1517-20.

2. Metcalfe SM. Mesenchymal stem cells and management of COVID-19 pneumonia. Med Drug Discov. 2020;5:100019.

3. Tang N, Li D, Wang X, Sun Z. Abnormal coagulation parameters are associated with poor prognosis in patients with novel coronavirus pneumonia. J Thromb Haemost. 2020;18(4):844-7.

4. Mehta P, McAuley DF, Brown M, Sanchez E, Tattersall RS, Manson JJ, et al. COVID-19: consider cytokine storm syndromes and immunosuppression. Lancet. 2020;395(10229):1033-4.

5. Jayaramayya K, Mahalaxmi I, Subramaniam MD, Raj N, Dayem AA, Lim $\mathrm{KM}$, et al. Immunomodulatory effect of mesenchymal stem cells and mesenchymal stem-cell-derived exosomes for COVID-19 treatment. BMB Rep. 2020;53(8):400-12.

6. Curley GF, Scott JA, Laffey JG. Therapeutic potential and mechanisms of action of mesenchymal stromal cells for Acute Respiratory Distress Syndrome. Curr Stem Cell Res Ther. 2014;9(4):319-29.

7. Matthay MA, Pati S, Lee JW. Concise review: mesenchymal stem (stromal) cells: biology and preclinical evidence for therapeutic potential for organ dysfunction following trauma or sepsis. Stem Cells. 2017;35(2):316-24.

8. Lopes-Pacheco M, Robba C, Rocco PRM, Pelosi P. Current understanding of the therapeutic benefits of mesenchymal stem cells in acute respiratory distress syndrome. Cell Biol Toxicol. 2020;36(1):83-102.

9. Laffey JG, Matthay MA. Fifty years of research in ARDS. Cell-based therapy for acute respiratory distress syndrome. Biology and potential therapeutic value. Am J Respir Crit Care Med. 2017;196(3):266-73.

10. Taghavi-Farahabadi M, Mahmoudi M, Soudi S, Hashemi SM. Hypothesis for the management and treatment of the COVID-19-induced acute respiratory distress syndrome and lung injury using mesenchymal stem cell-derived exosomes. Med Hypotheses. 2020;144:109865.

11. Yang K, Wang J, Wu M, Li M, Wang Y, Huang X. Mesenchymal stem cells detect and defend against gammaherpesvirus infection via the cGASSTING pathway. Sci Rep. 2015;5:7820.

12. Meisel R, Zibert A, Laryea M, Gobel U, Daubener W, Dilloo D. Human bone marrow stromal cells inhibit allogeneic T-cell responses by indoleamine 2,3-dioxygenase-mediated tryptophan degradation. Blood. 2004;103(12):4619-21.

13. Golchin A, Farahany TZ, Khojasteh A, Soleimanifar F, Ardeshirylajimi A. The clinical trials of mesenchymal stem cell therapy in skin diseases: an update and concise review. Curr Stem Cell Res Ther. 2019;14(1):22-33. 
14. Shu L, Niu C, Li R, Huang T, Wang Y, Huang M, et al. Treatment of severe COVID-19 with human umbilical cord mesenchymal stem cells. Stem Cell Res Ther. 2020;11(1):361.

15. Hashemian SR, Aliannejad R, Zarrabi M, Soleimani M, Vosough M, Hosseini $\mathrm{SE}$, et al. Mesenchymal stem cells derived from perinatal tissues for treatment of critically ill COVID-19-induced ARDS patients: a case series. Stem Cell Res Ther. 2021:12(1):91.

16. Meng F, Xu R, Wang S, Xu Z, Zhang C, Li Y, et al. Human umbilical cordderived mesenchymal stem cell therapy in patients with COVID-19: a phase 1 clinical trial. Signal Transduct Target Ther. 2020;5(1):172.

17. Xu X, Jiang W, Chen L, Xu Z, Zhang Q, Zhu M, et al. Evaluation of the safety and efficacy of using human menstrual blood-derived mesenchymal stromal cells in treating severe and critically ill COVID-19 patients: an exploratory clinical trial. Clin Transl Med. 2021;11(2):e297.

18. Lanzoni G, Linetsky E, Correa D, Messinger Cayetano S, Alvarez RA, Kouroupis D, et al. Umbilical cord mesenchymal stem cells for COVID-19 acute respiratory distress syndrome: a double-blind, phase 1/2a, randomized controlled trial. Stem Cells Transl Med. 2021;10(5):660-73.

19. Adas G, Cukurova Z, Yasar KK, Yilmaz R, Isiksacan N, Kasapoglu P, et al. The systematic effect of mesenchymal stem cell therapy in critical COVID-19 patients: a prospective double controlled trial. Cell Transplant. 2021;30:9636897211024942

20. Shi L, Huang H, Lu X, Yan X, Jiang X, Xu R, et al. Effect of human umbilical cord-derived mesenchymal stem cells on lung damage in severe COVID19 patients: a randomized, double-blind, placebo-controlled phase 2 trial. Signal Transduct Target Ther. 2021;6(1):58.

21. Liang B, Chen J, Li T, Wu H, Yang W, Li Y, et al. Clinical remission of a critically ill COVID-19 patient treated by human umbilical cord mesenchymal stem cells: a case report. Medicine (Baltimore). 2020;99(31):e21429.

22. Leng Z, Zhu R, Hou W, Feng Y, Yang Y, Han Q, et al. Transplantation of ACE2(-) mesenchymal stem cells improves the outcome of patients with COVID-19 pneumonia. Aging Dis. 2020;11(2):216-28.

23. Gorman E, Shankar-Hari M, Hopkins P, Tunnicliffe WS, Perkins GD, Silversides J, et al. Repair of acute respiratory distress syndrome by stromal cell administration (REALIST) trial: a phase 1 trial. EClinicalMedicine. 2021;41:101167

24. Aguiar FS, Melo AS, Araujo AMS, Cardoso AP, de Souza SAL, LopesPacheco M, et al. Autologous bone marrow-derived mononuclear cell therapy in three patients with severe asthma. Stem Cell Res Ther. 2020;11(1):167.

25. Matthay MA, Calfee CS, Zhuo H, Thompson BT, Wilson JG, Levitt JE, et al. Treatment with allogeneic mesenchymal stromal cells for moderate to severe acute respiratory distress syndrome (START study): a randomised phase 2a safety trial. Lancet Respir Med. 2019;7(2):154-62.

26. McIntyre LA, Stewart DJ, Mei SHJ, Courtman D, Watpool I, Granton J, et al. Cellular immunotherapy for septic shock. A phase I clinical trial. Am J Respir Crit Care Med. 2018;197(3):337-47.

27. Morales MM, Souza SA, Loivos LP, Lima MA, Szklo A, Vairo L, et al. Pilot safety study of intrabronchial instillation of bone marrow-derived mononuclear cells in patients with silicosis. BMC Pulm Med. 2015;15:66.

28. Harrell CR, Sadikot R, Pascual J, Fellabaum C, Jankovic MG, Jovicic N, et al. Mesenchymal stem cell-based therapy of inflammatory lung diseases: current understanding and future perspectives. Stem Cells Int. 2019;2019:4236973

29. Yan X, Fu X, Jia Y, Ma X, Tao J, Yang T, et al. Nrf2/Keap1/ARE signaling mediated an antioxidative protection of human placental mesenchymal stem cells of fetal origin in alveolar epithelial cells. Oxid Med Cell Longev. 2019;2019:2654910.

30. Sinclair KA, Yerkovich ST, Chen T, McQualter JL, Hopkins PM, Wells CA, et al. Mesenchymal stromal cells are readily recoverable from lung tissue, but not the alveolar space. Healthy Hum Stem Cells. 2016;34(10):2548-58,

31. Zhou Y, Yamamoto Y, Xiao Z, Ochiya T. The immunomodulatory functions of mesenchymal stromal/stem cells mediated via paracrine activity. J Clin Med. 2019;8(7):1025.

32. Weiss ARR, Dahlke MH. Immunomodulation by mesenchymal stem cells (MSCs): mechanisms of action of living, apoptotic, and dead MSCs. Front Immunol. 2019;10:1191.

33. Song N, Scholtemeijer M, Shah K. Mesenchymal stem cell immunomodulation: mechanisms and therapeutic potential. Trends Pharmacol Sci. 2020;41(9):653-64.
34. Galipeau J, Sensebe L. Mesenchymal stromal cells: clinical challenges and therapeutic opportunities. Cell Stem Cell. 2018;22(6):824-33.

35. Li W, Ren G, Huang Y, Su J, Han Y, Li J, et al. Mesenchymal stem cells: a double-edged sword in regulating immune responses. Cell Death Differ. 2012:19(9):1505-13

36. Deng Y, Zhang Y, Ye L, Zhang T, Cheng J, Chen G, et al. Umbilical cordderived mesenchymal stem cells instruct monocytes towards an IL10producing phenotype by secreting IL6 and HGF. Sci Rep. 2016;6:37566.

37. Luz-Crawford P, Djouad F, Toupet K, Bony C, Franquesa M, Hoogduijn MJ, et al. Mesenchymal stem cell-derived Interleukin 1 receptor antagonist promotes macrophage polarization and inhibits B cell differentiation. Stem Cells. 2016:34(2):483-92.

38. Ko JH, Lee HJ, Jeong HJ, Kim MK, Wee WR, Yoon SO, et al. Mesenchymal stem/stromal cells precondition lung monocytes/macrophages to produce tolerance against allo- and autoimmunity in the eye. Proc Natl Acad Sci USA. 2016;113(1):158-63.

39. Melief SM, Schrama E, Brugman MH, Tiemessen MM, Hoogduijn MJ Fibbe WE, et al. Multipotent stromal cells induce human regulatory T cells through a novel pathway involving skewing of monocytes toward antiinflammatory macrophages. Stem Cells. 2013;31(9):1980-91.

40. Schmidt A, Zhang XM, Joshi RN, lqbal S, Wahlund C, Gabrielsson S, et al. Human macrophages induce CD4(+)Foxp3(+) regulatory T cells via binding and re-release of TGF-beta. Immunol Cell Biol. 2016;94(8):747-62.

41. Chang Y, de Nadai P, Azzaoui I, Morales O, Delhem N, Vorng H, et al. The chemokine CCL18 generates adaptive regulatory $T$ cells from memory CD4+ T cells of healthy but not allergic subjects. FASEB J. 2010;24(12):5063-72.

42. Braza F, Dirou S, Forest V, Sauzeau V, Hassoun D, Chesne J, et al. Mesenchymal stem cells induce suppressive macrophages through phagocytosis in a mouse model of asthma. Stem Cells. 2016;34(7):1836-45.

43. de Witte SFH, Luk F, Sierra Parraga JM, Gargesha M, Merino A, Korevaar SS, et al. Immunomodulation by therapeutic mesenchymal stromal cells (MSC) is triggered through phagocytosis of MSC by monocytic cells. Stem Cells. 2018;36(4):602-15.

44. Abreu SC, Xisto DG, de Oliveira TB, Blanco NG, de Castro LL, Kitoko JZ, et al. Serum from asthmatic mice potentiates the therapeutic effects of mesenchymal stromal cells in experimental allergic asthma. Stem Cells Transl Med. 2019;8(3):301-12

45. Ghannam S, Pene J, Moquet-Torcy G, Jorgensen C, Yssel H. Mesenchymal stem cells inhibit human Th17 cell differentiation and function and induce a T regulatory cell phenotype. J Immunol. 2010;185(1):302-12.

46. Wang WB, Yen ML, Liu KJ, Hsu PJ, Lin MH, Chen PM, et al. Interleukin-25 mediates transcriptional control of PD-L1 via STAT3 in multipotent human mesenchymal stromal cells (hMSCs) to suppress Th17 responses. Stem Cell Rep. 2015;5(3):392-404.

47. Ge W, Jiang J, Arp J, Liu W, Garcia B, Wang H. Regulatory T-cell generation and kidney allograft tolerance induced by mesenchymal stem cells associated with indoleamine 2,3-dioxygenase expression. Transplantation. 2010;90(12):1312-20

48. Franquesa M, Mensah FK, Huizinga R, Strini T, Boon L, Lombardo E, et al. Human adipose tissue-derived mesenchymal stem cells abrogate plasmablast formation and induce regulatory B cells independently of T helper cells. Stem Cells. 2015;33(3):880-91.

49. Carter NA, Vasconcellos R, Rosser EC, Tulone C, Munoz-Suano A, Kamanaka $M$, et al. Mice lacking endogenous IL-10-producing regulatory $B$ cells develop exacerbated disease and present with an increased frequency of Th1/Th17 but a decrease in regulatory T cells. J Immunol. 2011;186(10):5569-79.

50. Davies LC, Heldring N, Kadri N, Le Blanc K. Mesenchymal stromal cell secretion of programmed death-1 ligands regulates $T$ cell mediated immunosuppression. Stem Cells. 2017;35(3):766-76.

51. Castro LL, Kitoko JZ, Xisto DG, Olsen PC, Guedes HLM, Morales MM, et al. Multiple doses of adipose tissue-derived mesenchymal stromal cells induce immunosuppression in experimental asthma. Stem Cells Transl Med. 2020;9(2):250-60.

52. Han Y, Li X, Zhang Y, Han Y, Chang F, Ding J. Mesenchymal stem cells for regenerative medicine. Cells. 2019;8(8):886.

53. Sadeghi S, Soudi S, Shafiee A, Hashemi SM. Mesenchymal stem cell therapies for COVID-19: current status and mechanism of action. Life Sci. 2020;262:118493. 
54. Matthay MA, Thompson BT, Read EJ, McKenna DH Jr, Liu KD, Calfee CS, et al. Therapeutic potential of mesenchymal stem cells for severe acute lung injury. Chest. 2010;138(4):965-72.

55. Savukinas UB, Enes SR, Sjoland AA, Westergren-Thorsson G. Concise review: the Bystander effect: mesenchymal stem cell-mediated lung repair. Stem Cells. 2016;34(6):1437-44.

56. Dimarino AM, Caplan Al, Bonfield TL. Mesenchymal stem cells in tissue repair. Front Immunol. 2013;4:201.

57. Shafiee A, Patel J, Lee JS, Hutmacher DW, Fisk NM, Khosrotehrani K. Mesenchymal stem/stromal cells enhance engraftment, vasculogenic and pro-angiogenic activities of endothelial colony forming cells in immunocompetent hosts. Sci Rep. 2017;7(1):13558.

58. Sun Z, Wang Y, Gong X, Su H, Han X. Secretion of rat tracheal epithelial cells induces mesenchymal stem cells to differentiate into epithelial cells. Cell Biol Int. 2012;36(2):169-75.

59. Paunescu V, Deak E, Herman D, Siska IR, Tanasie G, Bunu C, et al. In vitro differentiation of human mesenchymal stem cells to epithelial lineage. J Cell Mol Med. 2007;11(3):502-8.

60. Bernard O, Jeny F, Uzunhan Y, Dondi E, Terfous R, Label R, et al. Mesenchymal stem cells reduce hypoxia-induced apoptosis in alveolar epithelial cells by modulating HIF and ROS hypoxic signaling. Am J Physiol Lung Cell Mol Physiol. 2018;314(3):L360-71.

61. Zhou B, Zhong N, Guan Y. Treatment with convalescent plasma for influenza A (H5N1) infection. N Engl J Med. 2007;357(14):1450-1.

\section{Publisher's Note}

Springer Nature remains neutral with regard to jurisdictional claims in published maps and institutional affiliations.

- fast, convenient online submission

- thorough peer review by experienced researchers in your field

- rapid publication on acceptance

- support for research data, including large and complex data types

- gold Open Access which fosters wider collaboration and increased citations

- maximum visibility for your research: over $100 \mathrm{M}$ website views per year

At BMC, research is always in progress.

Learn more biomedcentral.com/submissions 